DESY 95-149

July 1995

Comments on a Recent Solution to Wightman's Axioms

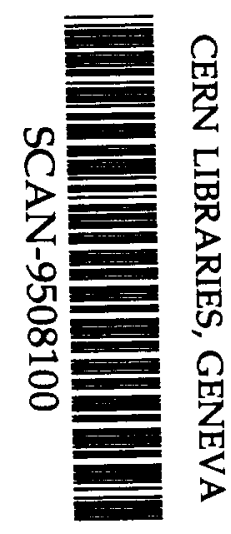

K.-H. Rehren

II. Institut für Theoretische Phvsik. Universität Hamburg

569533 
DESY 95-149

ISSN 0418-9833

July 1995

\title{
Comments on a recent solution to Wightman's axioms
}

\author{
K.-H. REHREN
}

II. Institut für Theoretische Physik, Universität Hamburg (GERMANy)

\begin{abstract}
:
A class of exact Wightman functionals satisfying all fundamental physical requirements in an arbitrary number of space-time dimensions, which bear the appearance of describing interacting fields, was recently constructed by C. Read [1]. It is shown here, that the construction can be considerably generalized, and that even the enlarged class belongs to the Borchers class of a system of generalized free fields.
\end{abstract}

\section{Introduction}

Ever since Wightman's formulation of the axioms [2] to be satisfied by the collection of $n$-point functions of local quantum fields, there has been a discomforting lack of models. Apart from models with polynomial interaction in two and three space-time dimensions, there are essentially only constructions based upon free fields and generalized free fields [3] available. These constructions involve Wick polynomials of derivatives of a given field, as well as so-called $p$ - and s-products [4] (i.e., pointwise products resp. sums of independent fields in different Hilbert spaces). Although one can easily produce nonvanishing truncated Wightman functionals, such models do not describe interacting particles.

Here is the well-known list of axioms for a hermitean scalar field, referring to the standard literature [2] for the precise formulation: Positivity and Hermiticity permit to reconstruct a Hilbert space containing the vacuum vector, and an (in general unbounded) hermitean field $\phi(f)$ on this Hilbert space whose vacuum correlation functions are given by the Wightman functional. Poincare Invariance of the Wightman functional ensures the invariance of a vacuum vector along with the Poincare covariance of the reconstructed field. The Spectrum Condition and Cluster Property ensure the positivity of the energy spectrum as well as the uniqueness of the vacuum. Finally, Locality expresses the local commutativity of the field $\phi(f)$.

In a recent paper [1], a new class of solutions was presented which satisfy all Wightman axioms in any number of space-time dimensions. The models are based on Feynman- 
like rules without at the same time being perturbative; instead, every $n$-point function is obtained as a sum over finitely many graphs. Some of the free input parameters of the models play a similar role as coupling constants in ordinary Feynman rules (although it will become clear in the course of this communication that they rather determine the structure of the field as a Wick polynomial), while the remaining parameters serve as appropriate cutoffs to ensure convergence of all sums and integrals involved. Unlike regulators in perturbative approaches, these cutoffs need not be removed in the end. The parameters comprise a smooth space-time cutoff function, a mass distribution in a finite mass interval, and a numerical limitation of the number of vertices.

In the present contribution, we intend to shed new light onto these models. It is found that the class of models [1] can be considerably extended by a generalization which essentially makes the "interaction polynomial" obsolete. We shall then reduce the entire construction to Wick powers of generalized free fields, and discuss aspects of the sharp mass limit of the extended class of models in comparison with the original class.

\section{A quick review of the original construction}

The structure of the n-point functions

$$
\mathcal{W}_{n}\left(f_{1}, \ldots, f_{n}\right) \equiv\left\langle\Omega, \phi\left(f_{1}\right) \cdots \phi\left(f_{n}\right) \Omega\right\rangle
$$

given in ref. [1] is the following (in somewhat schematic notation).

$$
\begin{aligned}
& \mathcal{W}_{n}\left(f_{1}, \ldots, f_{n}\right):=\sum_{\substack{\text { banded } \\
\text { graphs }}} \frac{1}{G_{\text {inter }} !} \int \prod_{e \in E_{\text {inter }}}\left(d g d p \theta\left(p^{0}\right)|\varphi(g p)|^{2}\right) \times \\
& \quad \times \prod_{i=1}^{n}\left[\hat{f}_{i}\left(k_{i}[p]\right) \times \frac{1}{G_{i, \text { dom }} !} \int \prod_{e \in E_{i, \text { dom }}}\left(d q \theta\left(q^{0}\right)|\varphi(q)|^{2}\right) \prod_{v \in V_{i}}\left(\nu ! a_{\nu} \delta_{v}(q, g p)\right)\right],
\end{aligned}
$$

where the sum extends over a class of (not necessarily connected) "banded graphs". A banded graph is a (possibly disconnected) graph which contains one connected subgraph ("band graph" or simply "band") for every field entry $\phi\left(f_{i}\right)$ in $(1)$ such that the sets $V_{i}$ of vertices of the $n$ bands are disjoint and exhaust all vertices of the full graph; every band has a distinguished "external" vertex of degree 1 . The internal vertices are of degree $2 \leq \nu \leq N$ for some finite number $N$. In each band the number $s_{i}$ of vertices is limited by some finite number $S$, and the vertices are labelled $1, \ldots s_{i}$. Inequivalent labellings of the vertices of the same abstract graph are considered as different banded graphs to be summed over. The banded graph has no external lines and no edges connecting a vertex with itself.

Edges which connect vertices of the same band will be called "domestic"; they are oriented from the vertex with lower ordinal number to the vertex with higher ordinal number; if a domestic edge connects to the external vertex of the band, then it may carry both orientations. [This restriction on the orientations of domestic edges in [1] 
seems not really to be necessary. We view it as another model input parameter.] Edges which connect vertices of different bands will be called "interband"; they are oriented from the band with lower ordinal number to the band with higher ordinal number. The integration rules are the following.

(i) Associated with every domestic edge $e \in E_{\mathrm{dom}}$ is a momentum variable $q \in$ $\mathbb{R}^{d, 1}$, to be integrated over with the measure $d q \theta\left(q^{0}\right)|\varphi(q)|^{2}$, where $\theta$ is the Heaviside step function for the energy, and $|\varphi(k)|^{2}=\mu\left(k^{2}\right) \cdot|\hat{\Theta}(k)|^{2}$ consists of a smooth mass distribution $\mu$ with support in the mass interval $m_{0}^{2} \leq k^{2} \leq m_{1}^{2}$ where $0<m_{0}<m_{1}$, and the square modulus of the Fourier transform of a real space-time cutoff function $\Theta(x)$ in the Schwartz space $\mathcal{S}\left(\mathbb{R}^{d+1}\right)$ (i.e., smooth and all derivatives decaying faster than any power of the arguments).

(ii) Associated with each interband edge $e \in E_{\text {inter }}$ are a momentum variable $p \in$ $\mathbb{R}^{d, 1}$ and a group variable $g \in L$ which runs over the four connected components of the full Lorentz group. These variables are integrated with the measure $d \mu(g, p)=$ $d g d p \theta\left(p^{0}\right)|\varphi(g p)|^{2}$ involving the right invariant Haar measure $d g$ on $L$. We notice that the variables $g$ enter only in the combination $g p$; thus effectively, the group integrations extend only over the Lorentz boosts and the time inversion in the rest frames of the momenta $p$. We may normalize to unity the redundant integral over the compact stabilizer group $O(d)$ of $p$, and therefore ignore it.

(iii) The combinatorial weights $G_{\text {inter }}$ ! and $G_{i, \text { dom }}$ ! are given by $\prod_{v, v^{\prime}} m\left(v, v^{\prime}\right)$ ! where $m\left(v, v^{\prime}\right)$ is the number of edges connecting the vertices $v$ and $v^{\prime}$, and the product extends over all pairs of vertices in different bands, and within the band $i$, respectively.

(iv) The integrand contains a "coupling constant" $\nu ! a_{\nu}$ for every internal vertex of degree $\nu$ along with a momentum conservation delta function for the momentum flow at that vertex given by the domestic momenta $q$ and the Lorentz transformed interband momenta $g p$.

(v) Finally, there contributes to the integrand a factor $\hat{f}_{i}\left(k_{i}\right)$ for every band, where $\hat{f}$ is the Fourier transform of the real test function $f \in \mathcal{S}\left(\mathbb{R}^{d+1}\right)$ and its value is taken at the momentum transfer $k=k[p]=\sum_{\text {out }} p-\sum_{\text {in }} p$. Here, the sums refer to all interband momenta flowing out of the band, resp. into the band at hand. It is a noteworthy fact that the momentum transfer of the field operators (involving the interband momenta $p$ ) is decoupled from the momentum flow within a graph (involving the transformed momenta $g p$ ).

The rapid decay of the integrand along with the momentum conservation delta functions guarantees that every integral in (2) converges absolutely. Due to the limitations $S$ of the number of vertices per band and $N$ of the degree of vertices, there are only finitely many different banded graphs, so the functional (2) is well defined. It is evident that it is translation invariant since the arguments of the test functions sum up to zero, $\sum_{i} k_{i}=0$, and that it is invariant under the orthochronous Lorentz group $L^{\uparrow}$ since for $\gamma \in L^{\uparrow}$ the change of the integrand can be absorbed into the change of variables $p \mapsto \gamma p$, $g \mapsto g \gamma^{-1}$ and $q \mapsto \gamma q$. The spectrum condition is satisfied since for every $j \leq n$, the sum of arguments $\sum_{i \leq j} k_{i}$ is a sum of interband momenta $p$ restricted to the forward light-cone. The uniqueness of the vacuum will become apparent later when we identify 
the Hilbert space.

The axiom with the most unprecedented realization in the new models is Locality. We shall limit ourselves here to the simple core of the exact but very tedious argument given in [1]. Namely, we shall discuss the commutativity of $\phi(f)$ and $\phi\left(f^{\prime}\right)$ when $f$ and $f^{\prime}$ are delta functions at space-like separated points $x$ and $x^{\prime} \in \mathbb{R}^{d, 1}$. Although these are not a priori admitted as test functions (the convergence argument given in [1] will fail for such functions), we may argue as follows for the validity of the simplified argument: Given the spectrum condition and Poincaré invariance, it is a standard result [2] that at the Jost points where all coordinates are space-like separated, the Wightman distributions are in fact functions. By the Reeh-Schlieder theorem 2, it is then sufficient to test the commutativity of $\phi(x)$ and $\phi\left(x^{\prime}\right)$ within Wightman functions at Jost points.

Consider therefore the change in the sum (2) when the field entries $\phi(x)$ at position $i$ and $\phi\left(x^{\prime}\right)$ at position $i+1$ are interchanged. Along with every banded graph contributing to $\mathcal{W}_{n}=\mathcal{W}_{n}\left(\ldots x, x^{\prime} \ldots\right)$ there corresponds a banded graph contributing to $\mathcal{W}_{n}^{\prime}=$ $\mathcal{W}_{n}\left(\ldots x^{\prime}, x \ldots\right)$ which differs only by the numbering of bands and therefore by the orientation of the interband edges extending from band $i$ to band $i+1$. Let there be $\sigma$ such edges in a given graph. The associated integration variables $g$ and $p$, here and later on collectively indicated by $(g, p)_{\sigma}$, enter the arguments of the test functions $f_{i}(k)=\exp -i k x$ and $f_{i+1}(k)=\exp -i k x^{\prime}$, the measure factors $\theta\left(p^{0}\right)|\varphi(g p)|^{2}$, and the momentum conservation delta functions. Due to Poincaré invariance, we are free to choose the Lorentz frame such that $x^{0}=x^{\prime 0}$. In this frame, each integral contributing to $\mathcal{W}$ is of the form

$$
\int \ldots \times \int \prod_{1}^{\sigma}\left(d g d p \theta\left(p^{0}\right)|\varphi(g p)|^{2} \exp \left(i \vec{p}\left(\vec{x}-\vec{x}^{\prime}\right)\right) \prod \delta_{v}\left(\ldots,(g p)_{\sigma}\right) \times \ldots\right.
$$

while the corresponding integral contributing to $\mathcal{W}^{\prime}$ is of the form

$$
\int \ldots \times \int \prod_{1}^{\sigma}\left(d g d p \theta\left(p^{0}\right)|\varphi(g p)|^{2} \exp \left(i \vec{p}\left(\vec{x}^{\prime}-\vec{x}\right)\right) \prod \delta_{v}\left(\ldots,(-g p)_{\sigma}\right) \times \ldots\right.
$$

where ... stands for further factors and dependences on other variables common to both contributions to $\mathcal{W}$ resp. $\mathcal{W}^{\prime}$, and independent of $(g, p)_{\sigma}$. By the change of integration variables $(g, p)_{\sigma} \mapsto(g T, P p)_{\sigma}$ where $T$ resp. $P$ are the time resp. space inversion in the given Lorentz frame, the integrands are transformed into each other (note that the cutoff function $\Theta$ is real, hence $\overline{\hat{\Theta}(k)}=\hat{\Theta}(-k)$, so $|\varphi(g p)|^{2}$ is invariant under $\left.g p \mapsto-g p\right)$. This establishes Locality, graph by graph.

Finally, Positivity of the Wightman functional (2) becomes manifest if one views every graph integral in $(2)$ as an operator product of integration kernels in the Fock space $\mathcal{F}(\mathcal{H})$ over the underlying Hilbert space $\mathcal{H}=L^{2}\left(L \times \mathbb{R}^{d, 1}, d \mu\right)$ such that every field operator $\phi(f)$ in $(1)$ is represented by a sum over such integral kernels: Every band subgraph with $\nu_{\text {out }}$ (resp. $\nu_{\text {in }}$ ) interband edges flowing out of (resp. into) it, and $\mu$ "bypassing" interband edges flowing from some lower band to some higher band, corresponds to a kernel interpolating from an $m$ - "particle" subspace $\mathcal{F}_{m}$ to an $n$-"particle" subspace $\mathcal{F}_{n}$ 
where $m=\nu_{\text {out }}+\mu$ and $n=\nu_{\text {in }}+\mu$. As a kernel, it is a function of $m$ sets of variables $(g, p)$ to be integrated (with the measure $d \mu$ ) with the arguments of a wave function in $\mathcal{F}_{m}$ ("annihilation part") and $n$ free sets of variables $(g, p)$ ("creation part"). It is given by the expression in square brackets in (2) as a function of the integration variables $(g, p)_{\text {out }}$ and $(g, p)_{\text {in }}$ associated with the out- and ingoing interband edges, while the bypassing edges contribute as delta function kernels $\delta\left(g, g^{\prime}\right) \delta\left(p-p^{\prime}\right) /|\varphi(g p)|^{2}$. (Note that every application of an integral kernel operator in $\mathcal{H}$ involves the integral measure $d \mu(g, p)=d g d p \theta\left(p^{0}\right)|\varphi(g p)|^{2}$, so one has not to worry about the denominators.)

A careful analysis of the combinatorics reveals that actually every field operator arises as sandwiched between the completely symmetrizing projection operator $\Pi=\bigoplus_{n} \Pi_{n}$ onto the symmetric Fock space $\mathcal{F}_{+}(\mathcal{H})=\Pi \mathcal{F}(\mathcal{H})$. Namely, let $(k)_{\text {in }}$ resp. $(k)_{\text {out }}$ denote a collection of ingoing resp. outgoing momentum variables, and let $K_{\nu_{\text {out }}}^{\nu_{\text {in }}}\left((k)_{\text {in }} ;(k)_{\text {out }}\right)$ be given by the domestic integrals summed over all band graphs with these variables assigned to the given number of in- and outgoing external lines:

$$
K_{\nu_{\text {out }}}^{\nu_{\text {in }}}\left((k)_{\text {in }} ;(k)_{\text {out }}\right)=\sum_{\substack{\text { band } \\ \text { graphs }}} \int \prod_{e \in E_{\text {dom }}}\left(d q \theta\left(q^{0}\right)|\varphi(q)|^{2}\right) \prod_{v}\left(\nu ! a_{\nu} \delta_{v}(q, k)\right)
$$

as read off eqn. (2). Clearly, $K_{\nu_{\text {out }}}^{\nu_{\text {in }}}$ vanish for $\nu_{\text {in }}+\nu_{\text {out }}>(N-1)^{S}$. Let furthermore

$$
\begin{aligned}
\Phi_{\nu_{\text {out }}, \mu}^{\nu_{\mathrm{in}}, \mu}\left((g, p)_{\mathrm{in}},(g, p)_{\mu} ;\right. & \left.(g, p)_{\mathrm{out}},(g, p)_{\mu}^{\prime}\right)= \\
& =\hat{f}(k[p]) K_{\nu_{\mathrm{out}}}^{\nu_{\mathrm{in}}}\left((g p)_{\mathrm{in}} ;(g p)_{\text {out }}\right) \otimes{(\text { delta kernels })^{\otimes \mu}}^{Q \mu}
\end{aligned}
$$

be integral kernels interpolating between $\mathcal{H}^{\otimes \nu_{\text {out }}+\mu}$ and $\mathcal{H}^{\otimes \nu_{\text {in }}+\mu}$ where $\hat{f}$ and $K_{\nu_{\text {out }}}^{\nu_{\text {in }}}$ are functions of the indicated in- and outgoing interband variables $k[p]=\sum_{\text {out }} p-\sum_{\text {in }} p$ and $g p$ only, and (delta kernels) ${ }^{\otimes \mu}$ stands for the delta functions in $\mu$ sets of bypassing interband variables $(g, p)_{\mu}$ as explained before.

Then $\phi(f)$ is represented by the integral kernels

$$
\phi(f)=\sum_{\nu_{\mathrm{in},}, \nu_{\mathrm{out}}, \mu} \Pi_{\nu_{\mathrm{in}}+\mu}\left(\frac{\sqrt{\left(\nu_{\mathrm{in}}+\mu\right) !\left(\nu_{\mathrm{out}}+\mu\right) !}}{\mu ! \nu_{\mathrm{in}} ! \nu_{\mathrm{out}} !} \Phi_{\nu_{\mathrm{out}}, \mu}^{\nu_{\mathrm{in}}, \mu}\right) \Pi_{\nu_{\mathrm{out}}+\mu}
$$

We shall refer to the functions (4) as the "reduced kernels". They are completely symmetric in either of their in- and outgoing sets of arguments. They encode the entire dependence of the model on the choice of the constants $a_{\nu}(\nu \leq N)$ and the number $S$ (limiting the number of graphs), as well as the previously mentioned restriction on the orientations of domestic edges. Since the remaining input parameters enter the integral measure of the Hilbert space $\mathcal{H}$, the model is now specified by the reduced kernels $K_{\nu_{\text {out }}}^{\nu_{\text {in }}}$ and the measure $d \mu$.

Since the assertion that the prescriptions (2) and (4-6) produce the same Wightman functional is the central claim of this section, and is essential for the rest of the paper, we formulate it as a technical Lemma.

Lemma. Let the fields o(f) be represented as integral kernels (4-6) on the symmetrized Fock space $\mathcal{F}_{+}(\mathcal{H})$. Let the vacuum vector $\Omega$ be represented by the 
number $1 \in \mathcal{F}_{0} \equiv \mathbb{C} \subset \mathcal{F}_{+}(\mathcal{H})$. Then the vacuum correlation of $\phi(f)$ coincide with eq. (2). More specifically, the sums over all band graphs in (4) reproduce the sum over all banded graphs in (2), while the numerical factors in (4) together with the combinatorial weights due to the symmetrizing projections conspire to reproduce the symmetry factors $\left(G_{\text {inter }} ! \prod_{i} G_{i, \mathrm{dom}} !\right)^{-1}$ in (2).

Proof: An $n$-point correlation is a sum over single band graphs contributing to every reduced kernel (4) and therefore to (5), and over single permutations contributing to the symmetrizing projections in (6). Every such contribution clearly corresponds to a banded graph contributing to the sum (2), and vice versa. However, the correspondence is not always one to one. What remains to be checked is that multiple counting and numerical factors produce the correct combinatorial weights as in eq. (2).

At this point, it is crucial that the sum (2) extends over all inequivalent labellings of the vertices of the banded graphs, while the sum (6) extends over all inequivalent labellings of the vertices and external lines of the band graphs.

Let us start with a two-point function and consider a banded graph $G$ contributing to (2) with its two band subgraphs $G_{1}$ and $G_{2}$. Let the vertices of $G_{1}$ be labelled as $v_{i}$, those of $G_{2}$ as $w_{j}$ (for this matter not distinguishing the external vertex of each band graph from its internal vertices), and let there $m_{i j}$ edges connect $v_{i}$ with $w_{j}$. Thus $\nu_{i}=\sum_{j} m_{i j}$ interband edges connect to $v_{i}$, and $\kappa_{j}=\sum_{i} m_{i j}$ interband edges connect to $w_{j}$. Let finally $\nu=\sum_{i} \nu_{i}=\sum_{j} \kappa_{j}$ denote the total number of interband edges of $G$. Apart from the domestic combinatorial weights (which are common to eq. (2) and eq. (4)), the banded graph enters eq. (2) with the weight $1 / G_{\text {inter }} !=\prod_{i j} 1 / m_{i j} !$. Assume for the moment that the symmetry groups of the vertices of $G_{1}$ and $G_{2}$ considered as abstract subgraphs of $G$, i.e., ignoring the labelling of vertices and orientation of edges, are trivial. This assumption is equivalent to the assumption that each latelling of the vertices gives rise to a different labelled band graph. Then there are $\nu ! / \prod \prod_{i} \nu_{i}$ ! inequivalent assignments of $\nu$ distinguished momenta $(k)=(g p)$ to the external linos of $G_{1}$, and similarly $\nu ! / \prod_{j} \kappa_{j}$ ! assignments of momenta to the external lines of $\rho_{2}$, cach giving rise to one term in the sums (4). Furthermore, each set of $\nu_{i}$ lines extending from $v_{i}$ can be partitioned in $\nu_{i} ! / \prod_{j} m_{i j}$ ! ways to join the vertices $w_{j}$ with multiplicities $m_{i j}$, and there is a similar number of partitions for the vertices $w_{j}$. Finally, each of the $m_{i j}$ ! contractions of $m_{i j}$ lines between $v_{i}$ and $w_{j}$ gives rise to the same banded graph. Thus the total number of contractions of band graphs contributing to (4) which give rise to the same banded graph $G$ equals

$$
\frac{\nu !}{\prod_{i} \nu_{i} !} \frac{\nu !}{\prod_{j} \kappa_{j} !} \prod_{i} \frac{\nu_{i} !}{\prod_{j} m_{i j} !} \prod_{j} \frac{\kappa_{j} !}{\prod_{i} m_{i j} !} \prod_{i j} m_{i j} !=\frac{(\nu !)^{2}}{G_{\mathrm{inter}} !} .
$$

Since the symmetrizing projection operator between the two kernels contributes a weight $1 / \nu$ ! for each permutation, and the numerical factors in (6) contribute another factor $(\sqrt{\nu !} / \nu !)^{2}=1 / \nu$ ! to the two-point function, we reproduce the combinatorial weight as in $(2)$.

Now let the vertices of the abstract band graphs $G_{i}$ possess some symmetry groups $S_{i}$ and let $S \subset S_{1} \times S_{2}$ be the symmetry group of the vertices of $G$. Then one may sum in 
(2) over all labellings of the internal vertices of $G_{1}$ and over all labellings of the internal vertices of $G_{2}$ independently, if one includes a correction factor $1 /|S|$ for overcounting of banded graphs. Similarly, one may sum in (4) over all labellings of internal vertices and over all labellings of external lines independently, if one includes a correction factor $\left|S_{i}\right|$ for each of the two kernels. On the other hand, the counting of inequivalent contractions of two band graphs with labelled vertices and external lines which give rise to the same banded graph with unlabelled interband edges provides an additional multiplicity factor $\left|S_{1} \times S_{2} / S\right|$ which cancels the correction factors for overcounting. We conclude that for two-point functions, the prescriptions (2) and (4-6) produce the same combinatorial weights.

Let us now turn to higher $n$-point functions. Repeating the previous reasoning with the obvious generalization, the total number of contractions of band graphs $G_{i}$ ( $i=$ $1, \ldots n$ ) contributing to (4) which give the same banded graph $G$ is found to exceed the expected weight $1 / G_{\text {inter }}$ ! by the factor $\prod_{i} \nu_{\text {in }}^{i} ! \nu_{\text {out }}^{i}$ ! where $\nu_{\text {in }}^{i}$ and $\nu_{\text {out }}^{i}$ are the number of in- and outgoing interband edges of $G_{i}$. This factor is cancelled by the corresponding factors in the denominator of the numerical factors in (6). The square root numerators of the latter (which arise twice each) are compensated by the weights $1 /(\nu+\mu)$ ! of each permutation within the projections $\Pi_{\nu+\mu}$ between two kernels, while the remaining factors $1 / \mu$ ! in the denominators of (6) are cancelled by the number of permutations of the sets of bypassing variables at each kernel. The discussion of symmetries of the vertices of band graphs also parallels the two-point case. This completes the proof of the Lemma.

Due to the Lemma, Positivity of the Wightman functional (2) is manifest. The orthochronous Lorentz group $L^{\uparrow}$ is represented on $\mathcal{H}$, and therefore on the Fock space by second quantization, by the tensor product of the natural action (on $p \in \mathbb{R}^{d, 1}$ ) and the right regular action (on $g \in L$ ). Since the measure is invariant under this action, the representation is unitary. For the same reason, functions of $k=g p$ represent Lorentz invariant elements of $\mathcal{H}$ or $\mathcal{F}_{+}(\mathcal{H})$, and the reduced kernels (4) commute with the action of $L^{\dagger}$. Consequently, the full kernels (5) and finally the fields (6) transform like scalar fields. Let us now close the list of arguments that eq. (2) fulfils all Wightman axioms.

It is clear that $\Omega$ is the only translation invariant vector in the reconstructed Hilbert space $\overline{\mathcal{D}_{\phi}}$, where $\mathcal{D}_{\phi}=\operatorname{span}\left(\prod \phi\left(f_{i}\right)\right) \Omega \subset \mathcal{F}_{+}(\mathcal{H})$ is the Wightman domain of the (unbounded) operators $\phi(f)$. $\phi$ is an hermitean field since

$$
\overline{K_{\nu_{\text {out }}}^{\nu_{\text {in }}}\left((k)_{\text {in }}:(k)_{\text {out }}\right)}=K_{\nu_{\text {int }}}^{\nu_{\text {out }}}\left((k)_{\text {out }} ;(k)_{\text {in }}\right) .
$$

Corollary [1]: The n-point distributions given by eq. (2) define a hermitean scalar local Wightman field.

\section{An enlarged class of local Wightman fields}

We make the following crucial observation. 
None of the arguments for Finiteness, Positivity, Translation Invariance, Lorentz Invariance and Spectrum Condition along with the Cluster Property is affected if one replaces the reduced integral kernels $K_{\nu_{\text {out }}}^{\nu_{\text {in }}}\left((k)_{\text {in }} ;(k)_{\text {out }}\right)$ given by $(4)$ as domestic integrals over band graphs by arbitrary smooth polynomially bounded functions of the respective in- and outgoing variables $k=g p$, and defines $\phi(f)$ by $(5)$ and $(6)$. Since the kernels only act between symmetrizing projections, these functions may be chosen symmetric in both of its two sets of arguments. Furthermore, the argument for Hermiticity of $\phi(f)$ is unaffected provided the reduced kernels satisfy the condition (7) above. These assertions are obvious except, maybe, in the case of Finiteness, for which we refer to the estimate (11) in the Lemma below.

Finally, the above simplified argument for Locality remains unaffected when in (3) the delta functions due to each band (integrated over the domestic variables) are replaced by the respective reduced kernels, provided

$$
K_{\mu}^{\nu+\sigma}\left((k)_{\nu} \cup(k)_{\sigma} ;(k)_{\mu}\right)=K_{\mu+\sigma}^{\nu}\left((k)_{\nu} ;(-k)_{\sigma} \cup(k)_{\nu}\right)
$$

where $U$ indicates the union of the respective collections of arguments. (The reduced kernels (4) have this symmetry.) Namely, when $K$ resp. $K^{\prime}$ refer to the reduced kernels due to the field entries $\phi(x)$ resp. $\phi\left(x^{\prime}\right)$, then in a typical contribution to $\mathcal{W}_{n}$ products of reduced kernels

$$
K_{\sigma+\kappa}^{-\lambda}\left(\ldots ;(g p)_{\sigma} \cup \ldots\right) K_{\mu}^{\prime \sigma+\nu}\left(\ldots \cup(g p)_{\sigma} ; \ldots\right)
$$

replace the delta functions in $(3 a)$, while in a corresponding contribution to $\mathcal{W}_{n}^{\prime}$,

$$
K_{\sigma+\mu}^{\prime \nu}\left(\ldots ;(g p)_{\sigma} \cup \ldots\right) K_{\kappa}^{\sigma+\lambda}\left(\ldots \cup(g p)_{\sigma} ; \ldots\right)
$$

replace the delta functions in $(3 b)$. As before in Sect. 2, the dots indicate dependences on other variables which are common to both contributions. With the same change of the integration variables $(g, p)_{\sigma}$ as before, the crossing symmetry (8) ensures $\mathcal{W}_{n}=\mathcal{W}_{n}^{\prime}$.

In view of the two conditions $(7)$ and (8), it is sufficient to specify a terminating (in order to guarantee temperedness of the ensueing distribution) sequence $F \equiv$ $\left(F_{\nu}\left(k_{1}, \ldots, k_{\nu}\right)\right)_{\nu}$ of smooth polynomially bounded symmetric functions satisfying

$$
\overline{F_{\nu}\left(k_{1}, \ldots, k_{\nu}\right)}=F_{\nu}\left(-k_{1}, \ldots,-k_{\nu}\right)
$$

(i.e., the Fourier transforms of real functions). We shall call functions satisfying (9) "hermitean". Then the reduced kernels

$$
K_{\mu}^{\nu}\left((k)_{\nu} ;(k)_{\mu}\right):=F_{\nu+\mu}\left((k)_{\nu} \cup(-k)_{\mu}\right)
$$

satisfy (7) and (8).

These kernels inserted into (5) and (6) define a manifestly finite hermitean scalar local Wightman field $\phi_{F}$. Due to the Lemma above, this class of fields extends the class presented in [1]. 
The preceding arguments apply also without substantial change to mixed Wightman functionals with field entries $\phi_{F^{(i)}}\left(f_{i}\right)$ specified by different collections $F^{(i)}$ of reduced kernels. The following conclusion is immediate.

Corollary: Every terminating sequence $F=\left(F_{\nu}\left(k_{1}, \ldots, k_{\nu}\right)\right)_{\nu}$ of (smooth polynomially bounded symmetric) hermitean functions defines, upon insertion of the reduced kernels (10) into (5) and (6), a hermitean scalar local Wightman field $\phi_{F}$ on the symmetric Fock space $\mathcal{F}_{+}(\mathcal{H})$. The fields $\phi_{F}$ associated with different collections $F$ are defined (as tempered operator-valued distributions) on the joint Wightman domain $\mathcal{D}=\operatorname{span}\left(\prod \phi_{F^{(i)}}\left(f_{i}\right)\right) \Omega \subset \mathcal{F}_{+}(\mathcal{H})$. They are relatively local with respect to each other.

We shall call a field $\phi_{F}$ "of order $\nu "$ if $F_{\nu} \neq 0$ and all other $F_{\mu}$ vanish. In the general case, $\phi_{F}$ is a finite sum over its components of order $\nu$.

The assignment $F \mapsto \phi_{F}$ is clearly linear (in the obvious sense for each component of order $\nu$ ). It is continuous in the following sense.

Lemma. The correlations of fields $\phi_{F^{(i)}}$ (of fixed order $\nu_{i}$ ) are bounded by

$$
\left|\left\langle\Omega, \phi_{F^{(1)}}\left(f_{1}\right) \cdots \phi_{F^{(n)}}\left(f_{n}\right) \Omega\right\rangle\right| \leq C_{\left(\nu_{i}\right)} \prod_{i}\left\|F^{(i)}\right\|
$$

where the constants $C_{\left(\nu_{i}\right)}<\infty$ depend on the test functions $f_{i} \in \mathcal{S}\left(\mathbb{R}^{d+1}\right)$, and

$$
\left\|F_{\nu}\right\|^{2}:=\sup _{m_{0}^{2} \leq m_{i}^{2}=p_{i}^{2} \leq m_{1}^{2}} \int\left(\prod_{i} d g_{i}\left|\varphi\left(g_{i} p_{i}\right)\right|^{2}\right)\left|F_{\nu}\left(g_{1} p_{1}, \ldots, g_{\nu} p_{\nu}\right)\right|^{2} .
$$

Thus, for each test function, the assignment $F \mapsto \phi_{F}(f)$ is weakly continuous on the joint Wightman domain $\mathcal{D}$.

Proof: Every correlation of field operators in the vacuum state is a finite sum over integrals of the form

$$
\int\left(\prod d p \theta\left(p^{0}\right)\right) \prod_{i=1}^{n} \hat{f}_{i}\left(k_{i}[p]\right) \int\left(\prod d g|\varphi(g p)|^{2}\right) \prod_{i=1}^{n} K^{(i)}\left((g p)_{\nu_{i}-\mu_{i}} ;(g p)_{\mu_{i}}\right)
$$

such that each momentum $g p$ enters precisely one of the reduced kernels $K^{(i)}=K_{\mu_{i}}^{(i) \nu_{i}-\mu_{i}}$ as an ingoing (creation) variable, and another one as an outgoing (annihilation) variable. The $g$-integrals over products of reduced kernels with the measure $d g|\varphi(g p)|^{2}$ can be estimated, by repeated use of the Cauchy-Schwarz inequality, by the product of the corresponding finite $L^{2}$-norms of the reduced kernels. The latter are functions of the involved $p^{2}$ only, and can in turn be estimated by the supremum over the mass interval $\left[m_{0}, m_{1}\right]$, i.e., the norms given by (12) common to all terms in the sum. After these crude estimates, the remaining $p$-integrals of the form $\int \prod\left(d p \theta\left(p^{0}\right)\right) \prod \hat{f}_{i}\left(k_{i}[p]\right)$ still converge absolutely due to the decay of the test functions [1]. Summing all these integrals yields the constants $C_{\left(\nu_{i}\right)}$. 


\section{Reduction to generalized free fields}

Let us now study some elementary cases, starting with fields of order 1 which we denote by $\varphi_{F}$ with $F_{1}(k)=F(k)$ a hermitean polynomially bounded smooth function. These comprise the fields in [1] when all "coupling constants" vanish, or when $S=0$, hence every band graph has only its external vertex of degree 1 and $F_{1}(g p)=1$. One finds that

$$
\left\langle\Omega, \varphi_{F^{\prime}}\left(f^{\prime}\right) \varphi_{F}(f) \Omega\right\rangle=\int d p \theta\left(p^{0}\right)\left(\int d g|\varphi(g p)|^{2} \overline{F^{\prime}(g p)} F(g p)\right) \cdot \hat{f}^{\prime}(p) \hat{f}(-p) .
$$

while all truncated higher $n$-point functions for fields of order 1 vanish. Thus, $\varphi_{F}$ is a generalized free field with mass distribution $\mu_{F}$ supported in the mass interval $m_{0}^{2} \leq p^{2} \leq m_{1}^{2}$

$$
\mu_{F}\left(p^{2}\right)=\int d g|\varphi(g p)|^{2}|F(g p)|^{2}
$$

The generalized free fields for different functions $F$ are in general not independent, i.e., they have correlations among each other. There are in fact countable many independent such fields. To see this, it is convenient to view a function $F(k)$ in the two-sheeted region $M=\left\{k \in \mathbb{R}^{d .1}: m_{0}^{2} \leq k^{2} \leq m_{1}^{2}\right\}$ as a family (labelled by the mass) of pairs of functions on velocity space $F_{ \pm}(m ; v):=F(k)$ where $k= \pm g_{v} p_{m}$ with $p_{m}=(m, \overrightarrow{0})$ a momentum vector in its rest frame and $g_{v} \in L^{\uparrow}$ the Lorentz boost by the velocity $v$. At each mass $p^{2}=m^{2}$, the value of the mass distribution in the mixed two-point function (13) is a scalar product in the space $L^{2}\left(\left\{v \in \mathbb{R}^{d}: v^{2}<1\right\} ; d \eta_{m}\right) \otimes \mathbb{C}^{2}$

$$
\int d g|\varphi(g p)|^{2} \overline{F^{\prime}(g p)} F(g p)=\frac{1}{2} \sum_{\Sigma=+,-\infty} \int d \eta_{m}(v) \overline{F_{\varepsilon}^{\prime}(m ; v)} F_{\varepsilon}(m ; v)
$$

with the measure $d \eta_{m}(v)=d^{d} v\left(1-v^{2}\right)^{-(d+1) / 2}\left|\varphi\left(g_{v} p_{m}\right)\right|^{2}$. The real linear space of hermitean functions corresponds to the +1 eigenspaces of the real linear symmetric involutive operator $\left(F_{+}, F_{-}\right) \mapsto\left(\overline{F_{-}}, \overline{F_{+}}\right)$. These eigenspaces possess countable orthogonal real bases of the form $\left(F_{+}^{\alpha}(m ; v), F_{-}^{\alpha}(m ; v)\right)$ with $F_{ \pm}^{\alpha}$ smooth and polynomially bounded in $u \equiv v / \sqrt{1-v^{2}}$ and $F_{-}^{\alpha}=\overline{F_{+}^{\alpha}}$. Since the measure $d \eta_{m}$ varies smoothly with $m$, the family of bases can be chosen to vary also smoothly with $m$. The real span of the functions $\left(k^{2}\right)^{n} F^{\alpha}(k):=m^{2 n} F_{ \pm}^{\alpha}(m ; v)$ at $k= \pm g_{v} p_{m} \in M$ is dense (in the topology $(12))$ in the space of hermitean polynomially bounded smooth functions on $M$. Since $\varphi_{k^{2} F}(f)=\varphi_{F}(-\square f)$, it follows from (11) that the countable family of independent generalized free fields $\varphi^{\alpha}=\varphi_{F^{\alpha}}$ has a dense Wightman domain in the reconstructed Hilbert space of all order 1 fields $\varphi_{F}$.

The next case is an order $\nu$ field with $F_{\nu}$ a constant function. One finds that

$$
\phi_{F}(f)=\frac{F_{\nu}}{\nu !}: \varphi_{1}^{\nu}:(f)
$$

is just a Wick power of the generalized free field $\varphi_{F=1}$ of order 1 . Similarly, if $F_{\nu}$ is the symmetrized tensor product of single variable hermitean functions

$$
F_{\nu}\left(k_{1} \ldots, k_{\nu}\right)=\sum_{\pi \in S_{\nu}} \prod_{i} F^{(i)}\left(k_{\pi(i)}\right)
$$


then the associated field $\phi_{F}$ of order $\nu$ is the Wick product

$$
\phi_{F}(f)=: \prod_{i} \varphi_{i}:(f)
$$

of the generalized free fields $\varphi_{i}=\varphi_{F^{(2)}}$ of order 1 . It is not very difficult to prove (16) and (18) by verifying that the combinatorics of the integral kernels produces precisely the products of two-point functions required by Wick ordering. Namely, every factor in (17) arising in a creation kernel will be eventually integrated with another such factor in an annihilation kernel, yielding a two-point function with mass distribution of the form (15), while the numerical factors in (6) cancel with the combinatorial factors due to symmetrization.

Now, every symmetric hermitean function $F_{\nu}$ in $\nu$ variables can be approximated (in the topology (12)) by real linear combinations of symmetrized tensor products of $\nu$ hermitean functions in one variable. We conclude:

Corollary: The fields $\phi_{F}$ of order 1 form a countable system of generalized free fields $\varphi$. The fields $\phi_{F}$ of order $\nu$ are approximated (in the sense of the Lemma of Sect. 3) by homogeneous real Wick polynomials :P( $\varphi)$ : of degree $\nu$ in the latter. The vacuum vector is cyclic in the Hilbert space $\overline{\mathcal{D}}$ with respect to the fields of order 1 , which consequently act irreducibly in $\overline{\mathcal{D}}$.

\section{Conclusion and discussion}

We can now apply the classical results in [5] to conclude that the new fields $\phi_{F}$, and in particular the fields constructed in [1] which are finite sums of fields $\phi_{F}$ of order $\nu$, $\nu \leq(N-1)^{S}$, belong to the Borchers class of the countable system of generajized free fields defined by their two-point functions (13). (As a reminder: the Borchers class of an irreducible field $\phi_{0}$ consists of all fields on the same Hilbert space which are relatively local with respect to $\phi_{0}$, and which are therefore automatically relatively local with respect to each other.) For fields with a sharp mass (such that the scattering matrix is defined), coincidence of the Borchers class implies coincidence of the scattering matrix $[5]$.

Let us therefore insert a remark concerning a limit of sharp mass $m_{1} \searrow m_{0}$ which is desirable for a particle interpretation. Note, however, that scattering aspects of fields without a sharp mass were also considered in, e.g., [6]. In the original class of models [1], the naive limit attained by sharpening the bare mass distribution $\mu\left(m^{2}\right)$ is severely obstructed since due to the momentum flow conservation involving both domestic and interband momenta there will always occur powers of several measure factors $|\varphi(q)|^{2}$ at the same argument in the integrands (e.g., for the domestic momenta associated with the edges connected to the external vertices in two-point functions; the problem will be aggravated whenever the coupling $a_{2} \neq 0$ ). In order to keep the highest of such powers regular in the sharp mass limit, all the lower powers must become suppressed, so the limiting Wightman functional will consist of products of two-point functions only and 
one ends up with a free field. Apart from this obstruction, every contribution from the cubic coupling $a_{3}$ will die out exactly as soon as $m_{1}<2 m_{0}$, due to momentum conservation at the triple vertex.

On the other hand, no such obstruction prevents us in the enlarged class of models $\phi_{F}$ from sharpening the measure $\mu\left(m^{2}\right)$ independently from the cutoff function $\Theta$ and the reduced kernels $F_{n}$. The singularity obstruction is absent since there are no domestic integrations, and the latter effect is absent since there is no momentum conservation at the kernels for the integration variables $p$ or $g p$. However, as we have seen, in the limit $\mu\left(m^{2}\right) \rightarrow \delta\left(m^{2}-m_{0}^{2}\right)$, the system of generalized free fields will become a countable family of independent Klein-Gordon fields $\varphi_{m_{0}}^{\alpha}$, and the limiting fields $\phi_{F}$ will be Wick polynomials therein.

To summarize the previous remarks, we have found that although the sharp mass limit is much more flexible within the enlarged class of models, the limiting fields will belong to the Borchers class of a countable family of massive free fields, and hence will not describe scattering [5]. Even if it remains to be clarified in which precise sense the corresponding conclusion is true for generalized free fields, in view of our results of Sect. 4 we do not share the optimism of ref. [1] that the new fields might describe interaction as long as the mass remains smeared. However, the approach of ref. [1], and in particular the surprising mechanism which restores locality upon integration over the "inner degrees of freedom" associated with the Lorentz group, might well contribute some new and interesting ideas to constructive quantum field theory.

Acknowledgments: The author is very much indebted to C. Read, who circulated his work prior to publication, and to D. Buchholz and J. Yngvason for valuable comments and pointing out some of the classical literature.

\section{References}

[1] C. J. Read: Quantum field theories in all dimensions, Univ. Cambridge (UK) preprint (1994), to appear in Commun. Math. Phys.

[2] R. F. Streater, A. S. Wightman: PCT, Spin and Statistics, and All That; Benjamin W. A., New York, Amsterdam (1964).

For a profound guide to more recent achievements of the theory see also:

R. F. Streater: Outline of axiomatic relativistic quantum field theory, Rep. Progr. Phys. 38, 771-846 (1975), especially Chapter 3.

[3] O. W. Greenberg: Generalized free fields and models of local field theory, Ann. Phys. (N.Y.) 16, 158-176 (1961).

[4] H.-J. Borchers: Algebraic aspects of Wightman field theory, in: R. N. Sen and C. Weil (eds.), Statistical Mechanics and Field Theory, Haifa Lectures 1971; Halstedt Press, New York (1972).

[5] H.-J. Borchers: Über die Mannigfaltigkeit der interpolierenden Felder zu einer kausalen S-Matrix, Nuovo Cim. 15, 784-794 (1960).

[6] A. L. Licht: A generalized asymptotic condition, Ann. Phys. (N.Y.) 34, 161-186 (1965). 